\title{
Sektion Gesundheit und Umwelt des BAG in Gefahr, Kinderaktionsplan Umwelt und Gesundheit gestrichen
}

\author{
Nicht nur die Sektion Gesundheit und Umwelt des BAG soll aus finanziellen \\ Gründen auf Ende 2007 geschlossen werden. Die Sparwut des Bundesrates \\ macht auch vor den Zukunftsaussichten der Kinder nicht halt: Er will auf den \\ Kinderaktionsplan Umwelt und Gesundheit verzichten. Die Ärztinnen und \\ Ärzte für Umweltschutz, das Forum für Praxispädiatrie und die Schweizerische \\ Gesellschaft für Pädiatrie betrachten diesen Entscheid als inakzeptabel. Ge- \\ meinsam lancierten die drei Organisationen deshalb eine Petition, die den \\ Bundesrat auffordert, seinen Fehlentscheid rückgängig zu machen.
}

Rita Molla

Andreas Biedermann ${ }^{b}$

a Geschäftsleiterin Ärztinnen und Ärzte für Umweltschutz

b Mitglied der Geschäftsleitung Ärztinnen und Ärzte für Umweltschutz
Korrespondenz: Dr. Rita Moll Hauptstrasse 52 CH-4461 Böckten r.moll@bluewin.ch
Der Bundesrat hat im April 2005 im Rahmen der Aufgabenverzichtsplanung beschlossen, auf Ende 2007 die Sektion Gesundheit und Umwelt des Bundesamtes für Gesundheit BAG zu schliessen, und minimiert damit die Bearbeitung von umweltbezogenen Gesundheitsthemen, obwohl es unbestritten ist, dass Umweltfaktoren die Gesundheit der Menschen beeinflussen und beeinträchtigen. In einigen Bereichen lassen sich die Gesundheitskosten beziffern. Allein durch die Luftverschmutzung des Verkehrs entstehen Gesundheitskosten von insgesamt 1,6 Milliarden Franken, 94\% davon sind dem Strassenverkehr anzulasten [1].

Es ist im öffentlichen Interesse, dass die $\mathrm{Zu}$ sammenhänge zwischen Umweltfaktoren und Gesundheit der Menschen aufgezeigt und Ansätze zur Vorsorge und Prävention umweltbedingter Krankheiten erarbeitet werden. Die Sektion Umwelt und Gesundheit des BAG hat in diesem Bereich wertvolle Arbeit geleistet. Sie hat umweltbezogene Gesundheitsthemen im Sinne der Vernetzung in andere Bundesämter und Politikbereiche eingebracht und die Bestrebungen des Bundes zur nachhaltigen Entwicklung stark mitgeprägt. Es wurden konkrete Aufgaben wie der Aktionsplan Umwelt und Gesundheit APUG erfüllt, Verhaltensempfehlungen zu umweltbedingten Gefahren für die Gesundheit (z. B. bei hohen Ozonwerten oder Hitzewellen) herausgegeben und vielfältige, brennende Fragen aus der Bevölkerung betreffend Umwelt und Gesundheit beantwortet. Der Nutzen des APUG wird mit einer Studie zu den volkswirtschaftlichen Auswirkungen untermauert. Seit 1997 - so das Er- gebnis - hat das Aktionsprogramm Umwelt und Gesundheit bei Kosten von 14 Millionen Franken im Bereich Gesundheit einen Nutzen von fast 20 Millionen Franken erwirkt [2].

Betroffen vom Sparbeschluss ist auch der Kinderaktionsplan für Umwelt und Gesundheit, auf den der Bundesrat verzichten will - entgegen den eingegangenen Verpflichtungen an der Ministerkonferenz für Umwelt und Gesundheit in Budapest.

Die europäischen Umwelt- und Gesundheitsminister haben sich am 25. Juni 2004 in Budapest verpflichtet, in ihren Staaten Kinder-UmweltGesundheits-Aktionspläne einzurichten. Ungeborene, Kinder und Jugendliche sind in ihren unterschiedlichen Entwicklungsphasen besonders schutzbedürftig und in ihrer körperlichen, seelischen und sozialen Entwicklung gegenüber verschiedensten Umwelteinflüssen empfänglich. Die Weltgesundheitsorganisation schätzt, dass in der Region Europa etwa ein Drittel der kindlichen Krankheiten auf Umwelteinflüsse zurückzuführen sind. Der «Aktionsplan zur Verbesserung von Umwelt und Gesundheit der Kinder in der Europäischen Region CEHAPE» [3] enthält vier Ziele: Zugang zu sauberem Wasser, Schaffung von sicheren, geschützten und unterstützenden Wohngegenden, Massnahmen gegen die Verschmutzung von Innen- und Aussenluft sowie die Verminderung der Exposition durch gefährliche Chemikalien. Ein Katalog von zielorientierten Massnahmen ist Bestandteil des CEHAPE. Bereits 2007 sollen die europäischen Staaten über ihre Aktionspläne und erste Umsetzungsschritte berichten. 
Die Ärztinnen und Ärzte für Umweltschutz, das Forum für Praxispädiatrie und die Schweizerische Gesellschaft für Pädiatrie begrüssen die Idee dieser Aktionspläne. Das physische Umfeld der Kinder bedarf dringend der Korrektur. Eine positivere Einstellung der Gesellschaft ihren schwächsten Gliedern gegenüber ist einzuleiten. Wir brauchen dringend mehr Raum zur kindergerechten Entwicklung. Es sind Verbesserungen notwendig etwa auf dem Gebiet der Luftreinhaltung, der Qualität der Innenraumluft und der Ernährung unter besonderer Berücksichtigung des Problems Adipositas.

Die drei Ärzteorganisationen betrachten den Verzicht auf die Sektion Umwelt und Gesundheit des BAG und auf den Kinderaktionsplan als inakzeptabel. Dies bedeutet eine erhebliche Schwächung der Bearbeitung von wichtigen Aufgaben und Herausforderungen im Kampf gegen umweltbedingte Krankheiten, speziell bei Kindern. Wertvolles Fachwissen und ein breites Netzwerk im Bereich Umwelt und Gesundheit gingen verloren, internationale Verpflichtungen würden nicht eingehalten. Aus gesundheitspolitischer und volkswirtschaftlicher Sicht ist dies nicht zu verantworten.
Die Ärztinnen und Ärzte für Umweltschutz, das Forum für Praxispädiatrie und die Schweizerische Gesellschaft für Pädiatrie fordern den Bundesrat mit einer Petition auf, auf seinen Entscheid vom April 2005 zurückzukommen und den gesundheitlichen Gefahren der Umweltbelastung das nötige Gewicht beizumessen. Die Gesundheit der Kinder als besonders empfindliche Gesellschaftsgruppe in unserer belasteten Umwelt muss gefördert werden: Die drei Ärzteorganisationen bitten die Kolleginnen und Kollegen, das Anliegen mit ihrer Unterschrift zu unterstützen.

Petitionsbogen sind zu beziehen über info@ aefu.ch oder www.aefu.ch.

\section{Literatur}

1 Externe Gesundheitskosten durch verkehrsbedingte Luftverschmutzung in der Schweiz. www.are.ch/ de/verkehr/kosten_nutzen/unterseite24/ unterseite11/index.html.

2 www.apug.ch/d/aktuell/Monat0511_0_1.php.

3 www.euro.who.int/document/e83338g.pdf, www.euro.who.int/document/che/gdoc08.pdf. 